\title{
El imperativo ético de la mejora moral
}

The ethical imperative of moral enhancement

\section{Francisco Lara Sánchez}

Profesor Titular de Universidad. Departamento de Filosofía I, Universidad de Granada. España

flara@ugr.es

\section{TECNOLOGÍAS DE MEJORA HUMANA: DEBATE ÉTICO E IMPACTO SOCIOCULTURAL MONOGRÁFICO COORDINADO POR MIGUEL MORENO (Universidad de Granada) y FRANCISCO LARA (Universidad de Granada)}

\section{RESUMEN}

El autor defiende que, bajo ciertas condiciones, el uso de sustancias para mejorar el comportamiento moral de los seres humanos no solo es éticamente aceptable, sino también exigible. Para argumentar a favor de esta tesis en el artículo se plantean tres objetivos. En primer lugar, mostrar hasta qué punto las intervenciones con serotonina y oxitocina podrían mejorar la moralidad de los seres humanos reduciendo la agresividad, incrementando la disposición a colaborar y aumentando la empatía. En segundo lugar, justificar la necesidad de ese tipo de intervenciones tanto para enfrentarnos a las grandes injusticias de nuestro mundo, como para garantizar la supervivencia de nuestra especie. Por último, responder a las objeciones de que el uso de tales sustancias podría conllevar efectos morales contraproducentes y de que enajenaría a los individuos mejorados.

\section{ABSTRACT}

The author argues that, under certain conditions, the use of substances to enhance the moral behavior of human beings is not only ethically acceptable but also mandatory. He intends to prove this argument by proposing three main goals. First, to demonstrate how interventions using serotonin and oxytocin could enhance human moraliy in order to decrease aggressiveness, increase willingness to cooperate and improve empathy. Second, to justify that such interventions are needed both to face the great injustices and to ensure the survival of our species. And third, to reply those objections saying that the use of such substances could lead to counterproductive effects and to alienate enhanced people.

\section{PALABRAS CLAVE}

mejora | serotonina | oxitocina | empatía | moralidad y neuroética

KEYWORDS

enhancement | serotonin | oxitocin | empathy | morality and neuroethics

\section{Introducción (1)}

Los últimos avances en neurología y neuroética nos han aportado mucha información sobre la base biológica del comportamiento moral y sobre el efecto que en este pueden producir ciertas intervenciones en la naturaleza humana. Ahora sabemos que es posible cambiar el carácter moral de una persona de modo que su comportamiento se ajuste a pautas de corrección ampliamente aceptadas. Esto se puede conseguir alterando su genética, por medio de estimulaciones electromagnéticas en su cerebro o administrando ciertas sustancias que afecten al sistema nervioso. Son técnicas que constituyen básicamente lo que se conoce como mejora moral. Aunque todas estas técnicas se encuentran en una fase muy inicial, solo aquella en que se usan sustancias neurales genera en la actualidad altas expectativas de, en un futuro no muy lejano, conocer y controlar los posibles efectos secundarios no deseados. Es por ello que esta técnica en particular suscita actualmente un amplio interés por averiguar si, llegado ese momento en que sea segura, sería éticamente aceptable. En este artículo me hago eco de ese interés y propongo una tesis para el debate. Defenderé que el uso seguro de sustancias para la mejora moral no solo es aceptable sino también exigible. Para ello parto de la idea de que necesitamos una ética nueva para enfrentarnos a los problemas y amenazas de la sociedad actual, global y tecnológicamente muy avanzada, y de que en absoluto podremos regirnos por esa ética si previamente no hemos modificado, con la ayuda de la biotecnología, nuestras cualidades morales. También respondo a objeciones sobre los efectos contraproducentes y la enajenación que podrían derivarse del uso de estas sustancias. 


\section{Serotonina y oxitocina}

La tendencia a la agresión tiene un fuerte componente biológico. Si el sistema del transmisor serotonérgico no funciona adecuadamente, el cortex orbitofrontal del cerebro, zona donde se regulan las emociones, recibe inadecuadas dosis de serotonina $y$, como consecuencia, a veces no se pueden controlar los impulsos violentos, ni regular las reacciones emocionales a la provocación. Por ello, si una persona con esta disfunción tomase un inhibidor selectivo de recaptación de serotonina (ISRS), sustancia usada para combatir trastornos psicológicos, podría ralentizar la absorción de serotonina y, de este modo, reducir su tendencia a agredir a los demás (Almeida 2005, Miczek 2007, Coccaro 2012 y Krakowski 2003). Por otro lado, la absorción de ISRS, con el consiguiente aumento del nivel de serotonina, también influye en el comportamiento moral incrementando la disposición de los sujetos a colaborar con los demás (Wood 2006 y Wallace 2007) y a, si estuviera en su mano, llevar a cabo repartos equitativos de bienes (Tse 2002).

Pero el conocimiento actual de la determinación biológica de conductas de relevancia moral no acaba ahí. También abarca la empatía, la disposición a ponerse en el lugar de los otros y, como consecuencia, a evitar su sufrimiento. Esta tiene su origen en los genes DRD4 y COMT (Hastings 2014) y está relacionada con la intervención en el sistema nervioso de la oxitocina (Rodrígues 2009, Reyes 2008, Singer 2008, Zak 2007 y Barraza 2010), una hormona que también funciona como neurotransmisor y que es responsable, en parte y entre otros muchos efectos, de la predisposición de algunos mamíferos al vínculo con la pareja y al cuidado de las crías (Hasting y otros 2014). Ha sido posible sintetizarla artificialmente y se ha comprobado que al suministrársela a los humanos, por vía nasal o intravenosa, estos mejoran algunas disposiciones o aptitudes importantes para la conducta social. Así, por ejemplo, se ha demostrado que la oxitocina puede ayudar a la adopción de la perspectiva ajena, pues facilita la identificación de estados emocionales observando fotos que solo muestran los alrededores de los ojos (Domes 2007), hace que el sujeto mantenga más la mirada hacia los ojos del otro (Guastella 2008), mejora el reconocimiento de las expresiones emocionales positivas (Marsh 2010), o ayuda a que los autistas comprendan mejor el lenguaje afectivo (Hollander 2007). Además, hay ya muchas evidencias científicas de que la motivación altruista de evitación del sufrimiento ajeno, característica de la empatía, tiene mucho que ver con la implicación de la oxitocina en los sistemas neurales que refuerzan el comportamiento social (Bartels 2004, Insel 2004 y Dolen 2013), y de que al administrarles esta sustancia a los seres humanos, estos se sacrifican más por los demás y se vuelven más confiados, recíprocos y generosos (Kosfeld 2005, Morhenn 2008 y Zak 2004, 2005 y 2007).

Ahora bien, antes de comenzar el análisis ético del uso de este tipo de sustancias, habría que hacer una matización importante. Aún cuando sea cierto, como decía más arriba, que estas intervenciones biológicas resultan menos problemáticas que las que utilizan técnicas genéticas y de estimulación electromagnética, tampoco están exentas de complicaciones. Ya que aún no se conoce con detalle la compleja participación de los elementos neurales en diferentes sistemas, el uso de la serotonina y de la oxitocina siempre puede acarrear efectos inesperados. Por ello, el análisis ético que de dicho uso llevaré a cabo en adelante estará condicionado a la certidumbre de que estas intervenciones no supongan serios riesgos para la salud de los seres humanos.

Supongamos, por tanto, que ya tenemos esa certeza sobre cómo administrar estas sustancias de modo seguro. ¿Sería aceptable entonces usarlas para hacernos más pacíficos, empáticos y colaborativos? Intuitivamente parece que sí. Al fin y al cabo eso es lo que persiguen padres, profesores, sacerdotes, responsables de las ONG o gobernantes cuando educan o sensibilizan, respectivamente, a hijos, alumnos, fieles, posibles afiliados o ciudadanos. Todos pretenden fomentar, con diversas estrategias persuasivas, una actitud más altruista y solidaria. Pero la educación y concienciación moral raramente consigue sus objetivos. Solo tenemos que fijarnos en las bajas tasas de voluntariado social y en los altos índices de desigualdad, corrupción y delincuencia. Cabría preguntarse entonces por qué no recurrir a sustancias inofensivas que nos permitan conseguir, de manera más eficiente y menos costosa (ahorrando todo el tiempo, dinero y sacrificio que requieren los procesos educativos y judiciales), el objetivo universal de que los seres humanos seamos mejores personas. En principio, poner reparos a estas intervenciones biológicas de mejora moral solo denotaría una evidente incoherencia con la mayoría de nuestros actos, intenciones, deseos e instituciones. 
Ahora bien, ¿por qué queremos mejorar moralmente a los seres humanos, sea biológica o educativamente? En términos muy generales, supongo que para hacer un mundo mejor, en el que haya mejor convivencia, menos sufrimiento y más justicia. Ahora bien, ¿es cierto que las intervenciones neurales aquí planteadas podrían ser un buen instrumento, mejor incluso que las educativas, para conseguir ese mundo mejor? Parece que no siempre.

Consideremos primero la serotonina. Señalé que uno de los positivos efectos de esta sustancia sería la reducción de la agresividad, y es cierto que esto podría librarnos del sufrimiento provocado por los violentos, pero también es verdad que este tipo de intervención podría dejarnos indefensos en situaciones que requieren el uso de la violencia para evitar, por ejemplo, que alguien abuse injustamente de otras personas (Chan 2011). Y algo parecido podría decirse respecto a la otra potencialidad mencionada de la serotonina: el incremento de la confianza y del sentido de justicia. En principio, el mundo podría mejorar si, gracias a esta sustancia, la gente está más dispuesta a colaborar con los demás para llevar a cabo actividades o establecer contratos e instituciones que beneficien a todos. Sin embargo, este incremento de la disposición a colaborar también puede resultar contraproducente. Si esta mejora solo se aplica a los individuos que lo soliciten, conseguiríamos que aquellos a los que les mueve sobre todo el interés propio, y que no solicitarían ser reformados, sientan menos presión para ser recíprocos con los demás. Estaríamos, por tanto, contribuyendo a extender la estrategia conductual del free-rider, la de aprovecharte de un bien colectivo sin cumplir con la parte que te correspondería en su producción o sin compensar a quien se haya sacrificado para que tú te beneficies. Pues ¿para qué va el free-rider a pagar con su esfuerzo y privaciones el beneficio obtenido de la ayuda ajena si los que le han ayudado lo han hecho incondicionalmente por su gran disposición a colaborar con los demás?

Estos efectos contraproducentes podrían evitarse, no obstante, si somos selectivos y cautelosos en el uso de ISRS. Así, con respecto a la agresividad, la administración de esta sustancia debería reservarse para casos de individuos que se muestran incapaces de controlar sus impulsos violentos. Para el resto de personas lo conveniente sería no realizar ninguna intervención neural al respecto y permitir así, como suele ser habitual, cierto grado de agresividad en situaciones extremas. De todos modos, incluso si se defendiera una generalización de este tipo de intervenciones, la objeción de que resultaría contraproducente carecería de sentido. Pues dado lo poco habitual de esas situaciones extremas, globalmente todos ganaremos más si la gente es, por lo general, menos agresiva. La fuerza de esta réplica reside en la evidencia de que si estar preparado para este tipo de situaciones extremas fuera algo positivo, deberíamos plantearnos intentar hacer más agresiva a la gente que por naturaleza no lo es, y ese no es el caso. Nos parece desaconsejable. Creemos que la forma adecuada de prevenir y resolver este tipo de situaciones que requieren agresividad es confiando en instancias sociales e institucionales creadas al efecto, y que han de tener, por ello, la exclusividad del uso de la fuerza y la violencia.

Por otro lado, en el caso de los efectos contraproducentes de utilizar la serotonina para incrementar la disposición a colaborar cabría decir que igualmente la clave estaría en administrarla adecuadamente. Hasta tal punto es una cuestión de regulación de su uso que, en la dosis correcta, la misma serotonina podría servir de antídoto contra ese efecto no deseado, que se le achaca, de fomentar la conducta del free-rider. Se ha comprobado que además de favorecer la confianza y la disposición a colaborar, el ISRS también incrementa el sentido de justicia. En diferentes estudios se observó que el sujeto al que se le había transfundido ISRS mostraba una mayor disposición a rechazar injustas ofertas de dinero; es decir, prefería que ninguno recibiera nada antes que aceptar que el compañero que había repartido el dinero se llevara mucho más que él (Crockett 2008).

Más problemáticos resultarían los efectos contraproducentes que podrían derivarse del uso de la oxitocina. Pues aunque, en principio, el uso de la oxitocina lleva a que la gente procure ayudar más a los demás, también hay estudios que demuestran lo contrario. Sostienen que esta sustancia solo surtiría efectos positivos en términos de empatía y habilidades sociales cuando el individuo al que se le administra interactúa con personas cercanas, familiares o fiables. Sin embargo, cuando se encuentra en una situación competitiva (Shamay-Tsoory 2009), de incertidumbre (Declerck 2010) y de ineficiencia institucional (Zak 2008), sus efectos de incremento de la empatía se reducirían considerablemente. Y podrían incluso ser inmorales cuando el individuo intervenido con oxitocina se relaciona con desconocidos, ante los que muestra una actitud muy defensiva (Declerck 2010 y Sheng 2013), que se plasma a veces en favoritismo con los de la misma raza (Sheng 2013 y De Dreu 2010 y 2011), en un excesivo conformismo con su grupo (Stallen 2012) e incluso en un comportamiento deshonesto, sin 
vacilación, para beneficiar a su propio grupo (Shalvi 2014).

Algunos podrían pensar que este efecto de parcialidad y favoritismo es consustancial a la empatía, que consiste en identificarse emocionalmente con el otro y que es originada por una sustancia, la oxitocina, que opera en las partes del cerebro relacionadas con las emociones. Aducirían que las emociones son desaconsejables para la perspectiva imparcial que ha de pretender la moralidad y que, por ello, en ética deberíamos prescindir de la empatía. Yo, sin embargo, creo que esta es una vía equivocada, pues olvida que la empatía es el prototipo de la moralidad. Adoptar un punto de vista moral implica, en un principio, transcender nuestra propia perspectiva y considerar también las creencias, intereses y sentimientos de todos los afectados por la acción. Esto es lo que propicia la empatía y por lo que esta acaba siendo la responsable de nuestra creencia en una obligación igualadora de ayudar a todo aquel que sufre con independencia de su singularidad, su grado de racionalidad o su capacidad de expresarse. Creo que en el desarrollo de esa predisposición empática a ponerse en el lugar del otro está la clave para evitar tanto el egocentrismo como un racionalismo exclusivo para el que las reglas no admiten excepciones, ni siquiera aquellas que resultan plenamente razonables cuando nos ponemos en el lugar del otro.

Ahora bien, para lo que sí debería servir la acusación de la tendencia a la parcialidad de la empatía, y de la oxitocina en cuanto potenciadora suya, es para advertirnos de que no debemos confiar en las reacciones afectivas como la única fuente de las actitudes morales. Como instancia motivacional muy determinada por las emociones es normal que la empatía pueda resultar distorsionada, haciendo oídos sordos a racionales exigencias como la de imparcialidad. Así, si nuestro objetivo es no perder de vista la imparcialidad, el incremento de la empatía debería ir siempre acompañado de correctores cognitivos y reflexivos que nos prevengan de arbitrarias discriminaciones. Ejemplos de estos correctores serían la solicitud de que los sentimientos de aprobación o desaprobación que subyacen a nuestros juicios morales tengan como objeto los tipos de acciones y no quiénes sean los afectado por las acciones (Prinz 2011: 228); la insistencia en el hecho de que los extraños comparten con uno creencias, valores y emociones complejas (Leyens 2001); la exigencia de que cada uno siempre esté dispuesto a justificar por qué considera moralmente a unos individuos, y no a otros; o la adquisición de toda aquella información extra que permita a quien empatiza generarse representaciones más precisas del estado mental del otro, especialmente si pertenece a otro grupo.

Algunos experimentos demuestran que la oxitocina podría ser eficiente también para, en determinados contextos y con una administración adecuada, estimular algunos de esos mecanismos correctivos de la tendencia a la parcialidad. Serviría, por ejemplo, para inclinar al individuo intervenido a dar más importancia a determinada información social, como la pertenencia del otro a un grupo estigmatizado (Tarrant 2009 y Decety 2010), que finalmente le llevase a empatizar más con él. Podría ser tan útil al respecto que algunos investigadores han defendido su potencial para conseguir incluso que los israelitas empatizaran más con los palestinos, principales víctimas del conflicto que les enfrenta (Shamay-Tsoory 2013).

Otros autores sugieren ir más allá aún y recurrir a la inteligencia artificial, a los avances tecnológicos en el campo de la recogida, cálculo y organización de información, para reducir o evitar esos efectos contraproducentes de las intervenciones biológicas aquí consideradas (Savulescu 2015). Se apuesta, en concreto, por la posible utilidad al respecto de integrar en el cerebro humano dispositivos tecnológicos con los que procesar la información recogida por múltiples sensores y bases de datos para después reconocer su importancia funcional en virtud del contexto. Con ellos también se podría alertar al agente de que ciertos aspectos, circunstanciales o no, de su propia fisiología, personalidad o entorno podrían estar afectando negativamente a la calidad de su juicio y conducta moral. Aspectos como, por ejemplo, si ha dormido o no suficientemente, el tiempo transcurrido entre las comidas, ciertos patrones de excitación, el grado de agotamiento en el autocontrol ante las tentaciones, los niveles hormonales o el ruido ambiental (Olsen 2010 y Montoya 2013). La tecnología computacional también podría aconsejar al agente sobre cómo obrar en cada momento si quiere ser coherente con sus valores y objetivos (Pontier 2012). Podría avisarle, incluso, de que ante ciertas decisiones particulares él puede estar dejándose llevar por ciertos prejuicios y datos irrelevantes; o de que simplemente no está considerando aspectos importantes del dilema o de la decisión que tiene ante sí.

En definitiva, los efectos de la serotonina y de la oxitocina con respecto a la moralidad varían según las circunstancias. A veces pueden ser contraproducentes y entonces deberíamos proceder bien suspendiendo la administración de la sustancia en cuestión, bien recurriendo al mismo tiempo a otro tipo 
de intervenciones, sean educativas, biológicas o incluso computacionales, que corrijan esos efectos no deseados. Corresponde ahora considerar, en caso de que pudiéramos usar exitosamente estas sustancias -dosificándolas y complementándolas con otras intervenciones según demandara cada situación- si sería correcto hacerlo.

\section{La mejora como una exigencia de la ética y de la supervivencia}

De lo expuesto hasta aquí concluyo que, siempre que se cumplan ciertas condiciones, no habría inconveniente ético en usar la serotonina y la oxitocina para mejorar moralmente a los seres humanos. Esas condiciones serían que este uso de sustancias no sea perjudicial para la salud y que los posibles efectos moralmente contraproducentes se corrijan, regulando su administración o complementándola con otro tipo de técnicas. Pero también, en todo momento, hemos dado por supuesto que las intervenciones de mejora deberían ser voluntarias. No obstante, esta condición de voluntariedad podría volverse problemática si, como voy a intentar mostrar a continuación, el coste de no universalizar la mejora moral fuera descomunal.

Para empezar, es evidente que los seres humanos no somos muy eficientes en nuestras decisiones morales. Nos preocupamos en exceso de los individuos más cercanos, en comparación con el desinterés que mostramos por el sufrimiento de extraños. También es verdad que nos afecta mucho más lo que está ante nosotros que lo lejano, incluso cuando lo lejano sea mucho más grave. Además, nuestro altruismo se bloquea cuando el sufrimiento ajeno se muestra con grandes números y cantidades, de modo que encajamos mejor la hambruna de millones de seres humanos que la petición de ayuda de una persona en nuestra puerta. Un altruismo que, por otro lado, se reduce aún más en virtud de dos ideas sobre la moralidad muy arraigadas en nosotros. Una, la de que somos mucho más responsables de lo que causamos directamente que de lo que simplemente permitimos que suceda. Y otra, la de pensar que con aquellos que no son parientes nuestros solo debemos colaborar si demuestran reciprocidad. Estas creencias morales componen el núcleo de una moral tradicional, conocida como del sentido común, que a su vez se va nutriendo de juicios morales basados en intuiciones inmediatas y en respuestas emocionales (Damasio 1994, Haidt 2001 y Singer 2005) que a posteriori intentamos justificar con razones ad hoc. Juicios que, a partir de la mencionada preferencia por los cercanos, esconden actitudes de favoritismo racial, nacional o cultural; que no tienen suficientemente en cuenta el sufrimiento mucho mayor de los extraños, y del que somos culpables simplemente por no hacer nada para evitarlo; que nos eximen de responsabilidad en pequeñas acciones que al ser sumadas a las de los demás pueden tener efectos catastróficos, como los del cambio climático... En definitiva, creo que hay razones contundentes para sostener que nuestra moral del sentido común dificulta la reflexión y posterior aceptación de una ética libre de prejuicios y argumentada desde una perspectiva global e imparcial desde la que poder enfrentarnos a las injusticias y amenazas de nuestro tiempo.

Esa moral del sentido común es tan limitadora precisamente por ser de otro tiempo; y está tan arraigada en nosotros porque es de un tiempo en el que nuestra especie ha vivido la mayor parte de su existencia. Es una estrategia conductual que, en términos evolutivos, ha resultado muy exitosa para la especie humana porque, tras un largo proceso de configuración, ha dado a los humanos las pautas para adaptarse a su entorno social. Un entorno de la especie humana que se ha mantenido básicamente uniforme durante mucho tiempo y que ha consistido en pequeñas comunidades en la que todos sus integrantes se conocían mutuamente e interaccionaban constantemente. En este entorno se necesitaban pautas de comportamiento para fomentar la cooperación grupal entre individuos interesados y prosperaron pautas muy practicables y basadas en la reciprocidad y la protección del grupo. Así, dichas pautas definían la responsabilidad en virtud de lo que uno hacía, más que por lo que dejaba de hacer; limitaban el destinatario de las obligaciones a aquellos del grupo de uno que correspondían a las invitaciones a colaborar y a pactar. Con tales pautas y en el marco de sociedades pequeñas en las que todos se conocían, la estrategia conductual del free-rider estaba abocada al fracaso.

El problema ahora es que el entorno ha cambiado drásticamente en poco tiempo y la moral, no. Vivimos en una sociedad totalmente distinta a aquella en la que ha vivido el ser humano la mayor parte del tiempo. La globalización que caracteriza a la sociedad actual hace que los efectos de nuestras omisiones sean reales y palpables (como en el caso del hambre mundial); que uno no pueda dejar de ser responsable de los resultados nefastos para todos (como el cambio climático) que se deriva de sus 
insignificantes acciones individuales; o que el comportamiento anónimo de los free-rider sea más difícil de detectar y, por tanto, de penalizar. Además, en esta nueva sociedad tecnológicamente avanzada, en la que el acceso por internet a la máxima información es universal, se multiplica considerablemente la capacidad de los grupos terroristas para afectar negativamente en la vida y el bienestar de todos. Pues bien, ante los graves retos de esta nueva sociedad para la convivencia y para la supervivencia de la especie, poco puede hacer la moral tradicional. Tampoco ayuda mucho el sistema liberal democrático de nuestras sociedades que, por girar siempre en torno a intereses nacionales y al derecho a la privacidad, se muestra estructuralmente incapaz de hacer frente a estas serias amenazas globales a las que nos estamos refiriendo (Persson 2011 y 2012: 12-41).

En conclusión, si tomamos como referencia un criterio ético según el cual importa mucho cuales sean las consecuencias de nuestras acciones (y omisiones), especialmente cuando estas conlleven el sufrimiento y la muerte de mucha gente, no podemos seguir operando con una moral tradicional incapaz de enfrentarse a los preocupantes problemas de la sociedad actual. Y dado el carácter inherente a los seres humanos de esa moral (y política) tradicional, cabe pensar que será de poca ayuda la concienciación y la educación moral, y que, por lo tanto, el recurso a sustancias como la serotonina y la oxitocina debería ser un imperativo ético.

\section{Mejora moral y alienación}

Pero las conclusiones éticas nunca son fáciles y la de que la mejora moral es un imperativo no es una excepción. En ética es importante que nos fijemos en las consecuencias globales e imparciales de nuestras acciones pero también es crucial la salvaguarda de la autonomía y de la integridad personal del agente. Por ello, alguien podría objetar al uso obligatorio de la serotonina y la oxitocina que este puede llevar a la enajenación de los individuos intervenidos. A continuación expongo la que para mí sería la mejor respuesta a cada una de las tres versiones que, a mi entender, podría adoptar esta objeción.

1. El individuo mejorado perdería identidad. Podría aducirse que al modificar el grado de empatía de alguien, su sentido de justicia o su agresividad, estaríamos modificando su personalidad y, por ello, sus señas de identidad. Sin embargo, esta crítica solo tendría sentido desde una concepción del yo que no es la predominante. Realmente no creemos que nazcamos con una forma de ser que haya que mantener siempre. Más bien pensamos que nos vamos haciendo a nosotros mismos conforme tenemos experiencias en la vida. Así, desde esta concepción del yo como autorrealización, las intervenciones biológicas de mejora podrían verse como un instrumento útil, más que un impedimento, para alcanzar esa identidad buscada. Si alguien usa estas sustancias para cambiar es porque, en virtud de una conclusión ética a la que él ha llegado, considera que su forma de ser no es la adecuada y que debe busca otra mejor.

2. El individuo mejorado perdería autonomía al convertirse en una marioneta. También podría objetarse que el individuo al ser mejorado dejaría de ser él mismo el responsable de sus decisiones. Es decir, que con la intervención biológica él acabaría siendo menos agresivo o egoísta no porque realmente él quiera, sino porque ha sido modificado para ello. Lo que se sostiene así es que la persona mejorada, al haber recurrido a intervenciones biológicas para cambiar su motivación, habría situado la fuente de sus decisiones y de su comportamiento en un lugar externo a él; que, en definitiva, habría dejado de ser propiamente un agente libre que toma sus propias decisiones para convertirse en una marioneta movida por los autores y por los efectos de las intervenciones a las que ha sido sometido. Sin embargo, podría aducirse que, en términos de autonomía, no habría diferencias significativas entre individuos moralmente mejorados por estas técnicas biológicas, aquí cuestionadas, y los mejorados por técnicas tradicionales, que nadie discute. La educación moral, al igual que otras formas tradicionales de concienciación o apoyo psicológico que permiten el desarrollo moral de los individuos, consiste en un proceso formativo que no se caracteriza por un método discursivo y autodidacta, aun cuando el objetivo sea que la persona educada acabe pensando por sí misma. Educar e intervenir biológicamente pueden ser consideradas como dos formas distintas, pero equiparables, de facilitar desde fuera la autonomía del sujeto, ayudando a este a superar las limitaciones cognitivas y motivacionales que les impiden conformar su conducta a esos valores o preferencias últimas a las que debería llegar por independiente y meditada deliberación (Dees 2011). 
3. El individuo mejorado perdería autonomía e identidad al convertirse en un autómata al servicio siempre del bien común. En esta crítica, a diferencia de la anterior, lo más significativo ya no sería el hecho de que con la mejora las decisiones se tomaran fuera de uno. Aquí se estaría objetando que aun en el caso de que el individuo consintiera plenamente, el resultado de las mejoras realizadas en él sería una personalidad tan exigente en términos morales que le llevaría a perder su propia identidad. Si es modificado para resultar más empático, por ejemplo, acabaría identificándose tanto con los demás que llegaría a concebir como incorrecta cualquier acción (u omisión) que no optimizara el bienestar humano, incluidas aquellas que responden a sus valores y proyectos personales.

Creo, sin embargo, que detrás de esta crítica se esconden dos errores. El primero sería el de suponer que solo se puede mejorar moralmente (en altruismo, sentido de la justicia, disposición a colaborar...) a costa de la libertad individual. Pero esto no es así. No tiene sentido decir que las personas más empáticas, por ejemplo las mujeres, que suelen serlo más que los hombres, tienen menos autonomía simplemente porque son más proclives a anteponer el bien colectivo al interés propio. Esto solo tendría sentido si pensamos la autonomía como simplemente hacer aquello que a uno más le conviene. Es más plausible concebirla, sin embargo, como un estado en que las acciones se ajustan a decisiones resultantes de un análisis racional de todas las opciones posibles de comportamiento, incluida la opción moral de sacrificar el interés propio por el colectivo. Y este estado de autonomía lo pueden poseer los agentes en diferente grado. Defender el uso de unas sustancias que hace que la gente esté más dispuesta a cooperar, a contener los impulsos agresivos y a ponerse en el lugar de los otros, siempre que eso sea corregido por mecanismos cognitivos y deliberativos que adviertan al agente de posibles excesos, errores y prejuicios en la deliberación, difícilmente puede considerarse un menoscabo de la autonomía. Más bien se trataría de lo contrario.

El segundo error es pensar que un incremento de la disposición a interesarse por el bien común, incluso a maximizarlo, conduce necesariamente a una estrategia conductual de permanente optimización del resultado de las acciones (que acaba negando la individualidad del agente). Esto no es así pues, aunque pueda resultar paradójico, pretender siempre hacer lo mejor suele ser en muchos casos la peor opción. Imaginemos un mundo en el que todos estuviéramos constantemente procurando el mayor bien para todos. Al estar calculando en todo momento qué hacer nos convertiríamos en meros autómatas al servicio del bien común, a los que el cálculo consecuencial les prohibiría dedicar tiempo y esfuerzo a sus propios proyectos. Nuestras vidas serían entonces monótonas y muy pobres en experiencias subjetivas gratificantes. No se podría decir que estuviéramos realmente en un mundo feliz. Es verdad que tampoco sería ideal ese otro mundo imaginario, aunque menos, en el que la gente se preocupara poco o nada por el bien común. En este se podría decir que los agentes disfrutarían de mucha autonomía, en ese sentido discutible de buscar cada uno su propio beneficio, pero a costa de una injusta situación de extrema desigualdad e insolidaridad. Por todo ello, si buscamos el bien para todos no conviene que los agentes se comporten como seres interesados, pero tampoco como incansables buscadores del bien común. Conviene que, sin renunciar a sus proyectos personales, se preocupen por los demás, a ser posible incorporando esta preocupación en la configuración de sus propios proyectos. Para ello, es evidente que sería de gran ayuda que se generalizara una mayor disposición a colaborar, a empatizar y a no ser agresivo. Pero no se olvide que estamos hablando de disposiciones a obrar así, no de la obligación de hacerlo siempre. Igual que alguien que ha nacido con esas disposiciones no es por ello un autómata moral, y a veces obra en contra de esas tendencias innatas, también podría hacerlo quien las consiga artificialmente recurriendo a la oxitocina y la serotonina.

\section{Conclusiones}

Si no conlleva efectos negativos para la salud, mejorar moralmente a los seres humanos con serotonina y oxitocina puede ser coherente con nuestro constante empeño, a nivel personal y social, de aumentar la solidaridad y respeto por los demás. En este empeño recurrimos a métodos educativos y de concienciación social pero, a la vista de los hechos, con resultados no muy esperanzadores. ¿Por qué no intentarlo entonces con intervenciones neurológicas? He defendido que la mejora moral resulta aún más imperiosa cuando nos percatamos de que nuestras predisposiciones morales, resultantes de un largo proceso evolutivo en un entorno social muy diferente al actual, suponen una seria limitación para enfrentarnos a injusticias como el hambre en el mundo o el cambio climático, y a serias amenazas como el terrorismo mundial. 
Es cierto que, en ciertos contextos, la serotonina y la oxitocina podrían conllevar efectos contraproducentes para la mejora moral. Por ello, antes de su uso deben estudiarse las posibilidades de regular su administración y de corregir esos efectos no deseados por medio de procesos educativos o de dispositivos que enriquezcan y mejoren nuestra capacidad de tomar decisiones.

He defendido también que en caso de poder llevarse a cabo esas intervenciones de mejora moral sin efectos secundarios indeseables y sin efectos contraproducentes, aquellas no supondrían una alienación para el intervenido. Más que cuestionarlas como impedimentos para el desarrollo autónomo de la persona, deberían ser concebidas como ayudas para superar las limitaciones de aquellos que no han tenido la suerte de nacer con predisposiciones que amplían la decisión más allá de la perspectiva puramente egocéntrica. Creo que utilizar sustancias para ello, después de corregir sus efectos perjudiciales, puede ser, en contra de lo que pudiera parecer a primera vista, un buen instrumento para incrementar la autonomía y el enriquecimiento personal.

\section{Notas}

1. En este artículo se recogen algunos de los resultados obtenidos en el desarrollo del proyecto de investigación "Ética y política de los avances biomédicos en mejora humana", con referencia FFI2012-32565 y financiado por el Ministerio de Economía y Competitividad del Gobierno de España.

\section{Bibliografía}

Almeida, R. M. (y otros)

2005 "Escalated Aggressive Behavior: Dopamine, Serotonin and GABA", European Journal of Pharmacology, $\mathrm{n}^{0} 526$.

Barraza, J.

2010 The Physiology of Empathy: Living oxytocin to empathic responding, Dissertation, Claremont Graduate University, Proquest.

Bartels, A. (y otros)

2004 "The neural correlates of maternal and romantic love", Neuroimage, n 21: 1155-1166.

Chan, S. (y J. Harris)

2011 "Moral Enhancement and Pro-Social Behaviour", Journal of Medical Ethics, n 37 (3).

Coccaro, E. F.

2012 "What is the nature of serotonergic abnormalities in human aggression?", Biological Psychiatry, $\mathrm{n}^{\circ}$ 72.

Crockett, M. J. (y otros)

2008 "Serotonin Modulates Behavioral Reactions to Unfairness", Science, nº 320.

Damasio, A.

1994 Descartes's error: Emotion, reason, and the human brain. G. P. Putnam's Sons.

De Dreu, C. (y otros)

2010 "The neuropeptide oxytocin regulates parochial altruism in intergroup conflict among humans", Science, $\mathrm{n}^{0}$ 328: 1408-1411.

2011 "Oxytocin promotes human ethnocentrism", Proceedings of the National Academy of Sciences, $\mathrm{n}^{\circ}$ 108: 1262-1266.

2010 "The blame game: the effect of responsibility and social stigma on empathy for pain", Journal of 
Cognitive Neuroscience, $\mathrm{n}^{\circ}$ 22: 985-997.

Declerck, C. (y otros)

2010 "Oxytocin and cooperation under conditions of uncertainty: the modulating role of incentives and social information", Hormones and Behavior, n 57: 368-374.

Dees, R.H.

2011 "Moral Philosophy and Moral Enhancements", AJOB Neuroscience, n 2.

Dolen, G. (y otros)

2013 "Social reward requires coordinated activity of nucleus accumbens oxytocin and serotonin", Nature, no 501: 179-184.

Domes, G. (y otros)

2007 "Oxytocin improves 'mind-reading' in humans", Biological Psychiatry, no 61: 731-733.

Guastella, A. J. (y otros)

2008 "Oxytocin increases gaze to the eye region of human faces", Biological Psychiatry, n 63: 3-5.

Haidt, J.

2001 "The Emotional Dog and Its Rational Tail: A Social Intuitionist Approach to Moral Judgment", Psychological Review, nº 108.

Hastings, P. D. (y otros)

2014, "The neurobiological bases of empathic Concern for Others" en K. Killen y J. Smetana, (eds.), Handbook of moral development, Psychology Press.

Hollander, E. (y otros)

2007 "Oxytocin increases retention of social cognition in autism", Biological Psychiatry, n 61: 498-503.

Insel, T. R. (y R. D. Fernald)

2004 "How the brain processes social information: Searching for the social brain", Annual Review ofNeuroscience, $\mathrm{n}^{\circ} 27$ : 697-722.

Kosfeld, M. (y otros)

2005 "Oxytocin increases trust in humans", Nature, n 435: 673-676.

Krakowski, M.

2003 "Violence and serotonin: Influence of impulse control, affect regulation, and social functioning", Journal of Neuropsychiatry and Clinical Neurosciences, $\mathrm{n}^{\circ} 15$.

Leyens, J. (y otros)

2001 "Psychological essentialism and the differential attribution of uniquely human emotions to ingroups and outgroups", European Journal of Social Psychology, $n^{\circ} 31:$ 395-411.

Marsh, A. (y otros)

2010 "Oxytocin improves specific recognition of positive facial expressions", Psychopharmacology, $\mathrm{n}^{\circ}$ 209: 225-232.

Miczek, K. A. (y otros)

2007 "Neurobiology of escalated aggression and violence", The Journal of Neuroscience, $\mathrm{n}^{\circ} 27$.

Montoya, E. R. (y otros)

2013 "Testosterone administration modulates moral judgments depending on second-to-fourth digit ratio", Psychoneuroendocrinology.

Morhenn, V. B. (y otros)

2008 "Monetary sacrifice among strangers is mediated by endogenous oxytocin release after physical contact", Evolution and Human Behavior, n² 29: 375-383.

Olsen, O. K. (y otros) 
2010 "The impact of partial sleep deprivation on moral reasoning in military officers", Sleep, $\mathrm{n}^{\circ} 33$ (8).

Persson, I. (y J. Savulescu)

2011 "Unfit for the Future? Human Nature, Scientific Progress, and the Need for Moral Enhancement", en J. Savulescu y otros (eds.), Enhancing Human Capacities, Blackwell.

2012 Unfit for the Future, Oxford University Press.

Pontier. M. A. (y J. F. Hoorn)

2012 "Toward machines tha behave ethically better than humans do", Proceedings of the $34^{\text {th }}$ International Annual Conference of the Cognitive Science Society, TX.

Prinz, J.

2011 "Is empathy necessary for morality?", en P. Goldie y A. Coplan (eds.), Empathy: Philosophical and psychological perspectives. New York, Oxford University Press: 211-229.

Reyes, T. (y J. Mateo)

2008 "Oxytocin and cooperation: Cooperation with non-kin associated with mechanisms for affiliation", Proceedings of the 2nd Annual Meeting of the North Eastern Evolutionary Psychology Society: 90-102.

Rodrigues, S. M. (y otros)

2009 "Oxytocin receptor genetic variation relates to empathy and stress reactivity in humans", Proceedings of the National Academy of Sciences of the United States of America, $\mathrm{n}^{\circ} 706: 21437-21441$.

Savulescu, J. (y H. Maslen)

2015 "Moral Enhancement and Artificial Intelligence: Moral Al", en Romportl y otros, Beyond Artificial Intelligence. Springer.

Shalvi, S. (y C. De Dreu)

2014 "Oxytocin promotes group serving dishonesty", Proceedings of the National Academy of Sciences USA, n 111: 5503-5507.

Shamay-Tsoory, S. (y otros)

2009 "Intranasal administration of oxytocin increases envy and schadenfreude (gloating)", Biological Psychiatry, nº 66: 864-870.

2013 "Giving peace a chance: Oxytocin increases empathy to pain in the context of the Israeli-Palestinian conflict", Psychoneuroendocrinology, no 38 (12): 3139-3144.

Sheng, F. (y otros)

2013 "Oxytocin modulates the racial bias in neural responses to others suffering", Biological Psychology, n० 92: 380-386.

Singer, $\mathrm{P}$.

2005 "Ethics and Intuitions", The Journal of Ethics, n 9.

Singer, T. (y otros)

2008 "Effects of oxytocin and prosocial behavior on brain responses to direct and vicariously experienced pain", Emotion, no 8: 781-791.

Stallen, M. (y otros)

2012 "The herding hormone: oxytocin stimulates in-group conformity", Psychological Science, $\mathrm{n}^{\circ} 23$ : 1288-1292.

Tarrant, M. (y otros)

2009 "Social categorization and empathy for outgroup members", British Journal of Social Psychology, $\mathrm{n}^{\circ}$ 48: 427-446.

Tse, W. S. (y A. J. Bond)

2002 "Serotonergic Intervention Affects Both Social Dominance and Affiliative Behaviour", 
Psychopharmacology, no 161.

Wallace, B. (y otros)

2007 "Heritability of Ultimatum Game Responder Behaviour", Proceedings of the National Academy of Sciences, $\mathrm{n}^{\circ} 104$ (40).

Wood, R. M. (y otros)

2006 "Effects of Tryptophan Depletion on the Performance of an Iterated Prisioner's Dilemma Game in Healthy Adults", Neuropsychopharmacology, nº 31.

Zak, P. J. (y otros)

2004 "The Neurobiology of Trust", Annals of the New York Academy of Sciences, nº 1032.

2005 "Oxytocin is associated with human trustworthiness", Hormones and Behavior, no 48: 522-527.

2007 "Oxytocin increases Generosity in Humans", Public Library of Science ONE, n 2 (11).

Zak, P.

2008 Moral markets: The critical role of values in the economy. Princeton University Press.

Gazeta de Antropología 